\title{
Focused Ion Beam Milled On-chip Resonator Nanostructures for Applications in Rare-Earth-Ion-Doped $\mathrm{Al}_{2} \mathrm{O}_{3}$ Active Waveguides
}

\author{
F. Ay, L.J. Kauppinen, J.D.B. Bradley, K. Wörhoff, R.M. de Ridder, and M. Pollnau \\ Integrated Optical MicroSystems (IOMS) Group, MESA+ Institute for Nanotechnology, \\ University of Twente, P.O. Box 217, 7500 AE Enschede, The Netherlands
}

\begin{abstract}
Reflection gratings on $\mathrm{Al}_{2} \mathrm{O}_{3}$ channel waveguides were defined by focused ion beam milling. Fabry-Perot microcavities were fabricated and improved performance upon annealing was demonstrated, making them viable candidates as resonators for on-chip waveguide lasers.
\end{abstract}

\section{INTRODUCTION}

The goal of realizing on-chip active optical components such as amplifiers and lasers has been driving the growing interest for research in the field of active integrated optical devices. Significant interest is currently dedicated to Si-technology compatible waveguide amplifiers and lasers.

We have concentrated our study on rare-earth-ion-doped amorphous $\mathrm{Al}_{2} \mathrm{O}_{3}$ layers grown on thermally oxidized silicon substrates. Reliable co-sputtering and channel waveguide fabrication methods, resulting in $\mathrm{Al}_{2} \mathrm{O}_{3}: \mathrm{Er}^{3+}$ channel waveguides with background losses as low as $0.21 \mathrm{~dB} / \mathrm{cm}$, have been developed $[1,2] . \mathrm{Er}^{3+}$-doped channel waveguides of thicknesses between 700-950 nm and waveguide widths of 4.0$8.0 \mu \mathrm{m}$ with varying $\mathrm{Er}^{3+}$ concentration were fabricated in order to investigate the net internal gain at $1533 \mathrm{~nm}$. The gain measurements were conducted by pumping at $980 \mathrm{~nm}$. A net gain of up to $2.0 \mathrm{~dB} / \mathrm{cm}$ was demonstrated at the emission peak of $1533 \mathrm{~nm}$ [3]. The experimental and simulated results for maximum internal net gain per unit length as a function of $\mathrm{Er}$ concentration are in good agreement (see Fig. 1).

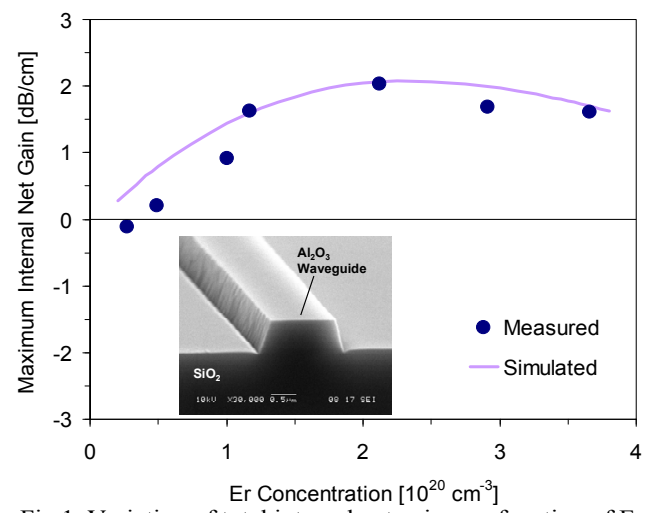

Fig.1. Variation of total internal net gain as a function of $\mathrm{Er}$ concentration [3]. The inset shows a scanning electron micrograph of a channel waveguide.

\section{Focused ION BEAM NANOSTRUCTURING}

In order to realize fully integrated waveguide lasers based on this technology on-chip resonator structures are to be defined. In this report we will focus on achieving integrated waveguide Fabry-Perot (F-P) microcavities. For realizing the required reflection gratings development of a high-quality micro- and nanostructuring process is of crucial importance.

An emerging technology which enables rapid and flexible nanometer-size feature definition is focused ion beam (FIB) milling. Since the method involves physical removal of material by a beam of $\mathrm{Ga}^{+}$ions, the technique can be adapted and optimized for almost any material system.

A Nova 600 dual-beam FIB machine was used for the definition of sub- $\mu \mathrm{m}$-period surface-relief reflection gratings on $\mathrm{Al}_{2} \mathrm{O}_{3}$ channel waveguides. The acceleration voltage was set to $30 \mathrm{kV}$ and the milling current was chosen to be $48 \mathrm{pA}$. The thickness of the $\mathrm{Al}_{2} \mathrm{O}_{3}$ channel waveguides was $\sim 550 \mathrm{~nm}$ and the grating structures were milled by $\sim 200 \mathrm{~nm}$ (Fig. 2). The length of each grating was $\sim 47.5 \mu \mathrm{m}$. The cavity length was varied between $100-450 \mu \mathrm{m}$ and waveguides with widths between 2.8 and $3.4 \mu \mathrm{m}$ were used.

A study toward minimization of redeposition effects was performed in order to obtain uniform and smooth sidewalls of the grating structures. The gratings were realized using a predefined mask file (stream file) that contains milling time, pixel information, and pixel sequence for the desired geometry.

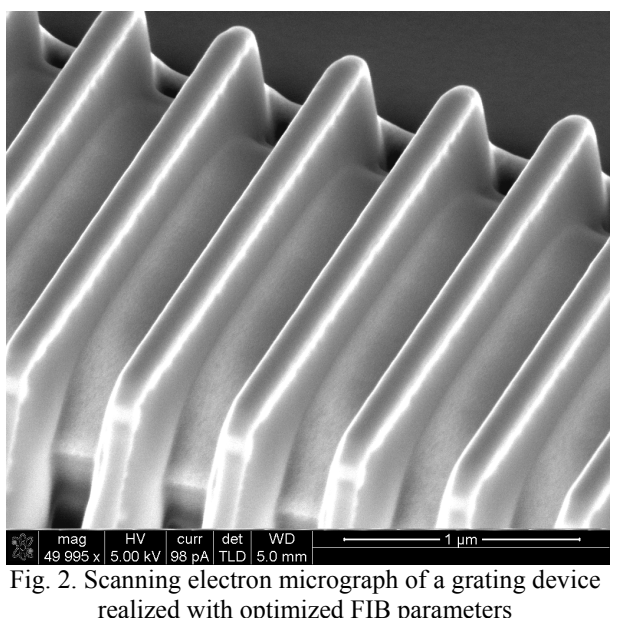


By optimizing FIB milling parameters such as ion current, dwell time, loop repetitions, scanning strategy, and applying a top metal layer for reducing charging effects during milling and improving sidewall definition, reflection gratings on $\mathrm{Al}_{2} \mathrm{O}_{3}$ channel waveguides with smooth and uniform sidewalls were fabricated.

\section{OPTICAL CHARACTERIZATION}

In order to assess the optical properties of the grating structures optical Fabry-Perot microcavities with two identical gratings were defined. Measured F-P transmission resonances in the 1540-1577-nm wavelength range are plotted in Fig. 3 (black dashed line). Values of finesse $F$ and cavity length $L_{C}$ were obtained through a fit to the experimental data. For a given cavity index $n_{C}$, the total effective overall distributed-loss coefficient $\alpha_{T}$ is related to $F$ by [4]

$$
F=\frac{\pi \times e^{-\alpha_{T} n_{C} L_{C} / 2}}{1-e^{-\alpha_{T} n_{C} L_{C}}} .
$$

The total loss $\alpha_{T}$ is an effective overall distributed-loss coefficient and is given by

$$
\alpha_{T}=\alpha_{C}+\frac{1}{2 n_{C} L_{C}} \times \ln \frac{1}{R^{2}},
$$

where $\alpha_{C}$ is the cavity loss, the quantity $1 /\left(2 n_{C} L_{C}\right) \times\left(\ln 1 / R^{2}\right)$ represents the loss coefficient attributed to the two DBR gratings, and $R$ is the reflectance.

The resulting resonator loss was found to be $6.9 \mathrm{~dB}$ with corresponding DBR reflectivity of $10 \%$ and finesse of 1.05 . The loss value includes scattering, out-of-plane losses, and the losses arising due to $\mathrm{Ga}^{+}$ion implantation during the FIB milling process. The most detrimental factor is likely to be the implantation of $\mathrm{Ga}^{+}$ions. In order to test this hypothesis we annealed the sample at $600{ }^{\circ} \mathrm{C}$ for 17 hours in $\mathrm{N}_{2}$ atmosphere. The experimental and calculated transmission spectra of the annealed F-P cavity are plotted in Fig. 3. An increase in reflectivity is expected to reduce the penetration depth of the mode into the DBR grating region and reduce the effective cavity length and thereby result in increase of the FSR. In accordance with this, an increase of the FSR from $3.92 \mathrm{~nm}$ to

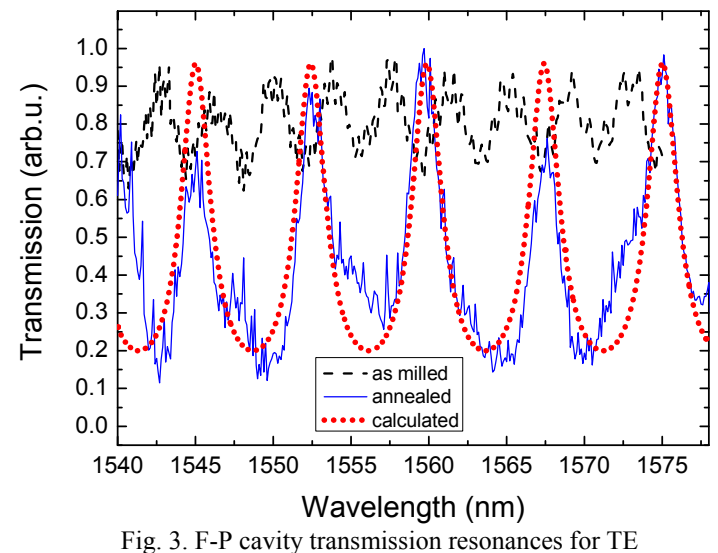

Fig. 3. F-P cavity transmission resonances for TE polarization of the as-milled and annealed cavity

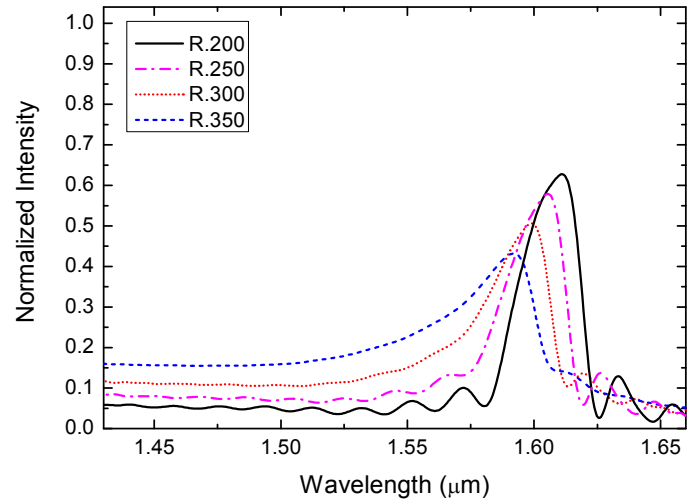

Fig. 4. 3D finite-difference time-domain simulation results of reflectivity for various milling depths of the grating structure

$7.53 \mathrm{~nm}$ after annealing was observed. The resonator finesse increased 3-fold from 1.05 for the as-milled cavity to 3.1. The resonator losses were reduced from $6.9 \mathrm{~dB}$ to $2.8 \mathrm{~dB}$. The reflectivity of the DBR gratings increased to $40 \%$, which is the lower bound for the DBR reflectance.

In order to estimate the contribution of $\mathrm{Ga}^{+}$ion implantation and consecutive annealing to the overall optical losses we performed a 3D finite-difference time-domain simulations (Fig. 4) using the Meep software package [5]. Due to grid-size considerations, the period of the simulated structure was chosen to be $550 \mathrm{~nm}$, resulting in a slight shift of the reflection peak to higher wavelengths. The simulation results suggest that, although minimized, the effect of $\mathrm{Ga}^{+}$implantation is still present, reducing the reflectivity by $5-10 \%$. Further simulations indicate the potential to increase the grating reflectivity up to $90 \%$. Therefore, even with remaining effects of $\mathrm{Ga}^{+}$ implantation, FIB patterned grating structures are viable candidates for use in on-chip waveguide lasers.

\section{CONCLUSIONS}

By optimizing FIB milling parameters reflection gratings on $\mathrm{Al}_{2} \mathrm{O}_{3}$ channel waveguides with smooth and uniform sidewalls were fabricated. F-P microcavities were defined and used to assess their optical performance. Reduction of optical losses by thermal annealing was demonstrated and the feasibility of using FIB patterned resonator structures for cavities in on-chip waveguide lasers in rare-earth-ion-doped materials was shown.

\section{REFERENCES}

[1] K. Wörhoff, J. D. B. Bradley, F. Ay, D. Geskus, T. Blauwendraat, and M. Pollnau, "Reliable low-cost fabrication of low-loss $\mathrm{Al}_{2} \mathrm{O}_{3}: \mathrm{Er}^{3+}$ waveguides with 5.4-dB optical gain," IEEE J. Quantum Electron. 45, 454-461 (2009).

[2] J. D. B. Bradley, F. Ay, K. Wörhoff, and M. Pollnau, "Fabrication of lowloss channel waveguides in $\mathrm{Al}_{2} \mathrm{O}_{3}$ and $\mathrm{Y}_{2} \mathrm{O}_{3}$ layers by inductively coupled plasma reactive ion etching," Appl. Phys. B 89, 311-318 (2007).

[3] J. D. B. Bradley, L. Agazzi, D. Geskus, F. Ay, K. Wörhoff, and M. Pollnau, "Gain bandwidth of $80 \mathrm{~nm}$ and $2 \mathrm{~dB} / \mathrm{cm}$ peak gain in $\mathrm{Al}_{2} \mathrm{O}_{3}: \mathrm{Er}^{3+}$ optical amplifiers on silicon," submitted.

[4] M. W. Pruessner, T. H. Stievater, and W. S. Rabinovich, "Integrated waveguide Fabry-Perot microcavities with silicon/air Bragg mirrors," Opt. Lett. 32, 533-535 (2007).

[5] A. Farjadpour, D. Roundy, A. Rodriguez, M. Ibanescu, P. Bermel, J. D. Joannopoulos, S. G. Johnson, G. Burr, "Improving accuracy by subpixel smoothing in FDTD," Opt. Lett 31, 2972-2974 (2006). 